\title{
THERMAL HYDRODYNAMIC FLUCTUATIONS NEAR THE RAYLEIGH-BENARD INSTABILITY
}

\author{
V. P. Lesnikov \\ Odessa State Polytechnic University, Department of Physics, UA-270044, Odessa, Ukraine
}

(Received November 5, 1996)

\begin{abstract}
The equivalence of Langevin's and Einstein's approaches for description of the thermal hydrodynamic fluctuations is shown. The erroneousness of the analogy of convective instability with phase transitions based on the use of Landau-Lifshitz fluctuating forces is pointed out.

Key words: fluctuations, fluctuating forces, fluctuation-dissipation theorem, Landau-Lifshitz fluctuating forces, instability, phase transitions.
\end{abstract}

PACS number(s): 47.20.-K

\section{INTRODUCTION}

In the late $60 \mathrm{~s}$ Uhlenbeck in the course of lectures devoted to the fundamental problems of statistical mechanics advanced the idea that near the threshold of hydrodynamic stability hydrodynamic fluctuations should be enhanced similar to thermodynamic fluctuations with the approach to the point of a second-order phase transition, leading to the critical opalescence, in particular [1].

Uhlenbeck suggested the Langevin description of fluctuations with the use of the fluctuating forces obtained by Landau and Lifshitz for equilibrium unlimited liquid [2] as a fluctuation source, i.e. thermal fluctuations were meant.

In this spirit Zaitsev and Shliomis examined the behaviour of hydrodynamic fluctuations near the convective Rayleigh-Benard instability [3] and came to the conclusion about the indefinite growth of integrated intensities of fluctuations, confirming thereby the Uhlenbeck's idea.

The opposite results on the basis of Einstein's approach consisting in averaging the initial conditions in the found fluctuation time-dependence, were obtained in [4].

For the single-time correlation functions equilibrium estimates were used in [4] with the corrections on temperature gradient obtained from kinetic theory by Hinton [5]. It was supposed that non-equilibrium Hinton's corrections exhaust all the first order corrections on the temperature gradient to the basic equilibrium approximation defined by some average temperature of the liquid layer. It will be shown below, that the significant corrections reflecting non-equilibrium (as a matter of fact, local-equilibrium) are corrections following from localequilibrium estimates. As to the kinetic corrections, there was no need in their use within the hydrodynamic limit.

Nevertheless it is already in the equilibrium approximation which is equivalent to the Langevin approach with the constant temperature in the sources of fluctuations Einstein's approach gives results contradicting to Uhlenbeck's assumption. The main result of [4] is that the fluctuation intensity remains constant while ap- proaching the Rayleigh-Benard instability point, and peculiarities consist only in narrowing the fluctuation line corresponding to the critical mode in spectra.

However this point of view is not common. The authors of subsequent works on hydrodynamic fluctuations near the convective instability used the Lagevin approach with the Landau-Lifshitz fluctuating forces. Since the intensity of the latter is fixed it was supposed that the intensity of fluctuation sources does not depend on the proximity of the threshold of stability. As a result in [610], as well as in [3], the conclusion was made about the unlimited growth of fluctuations and the analogy with phase transitions was formulated.

The same tendency was continued elsewhere [11], see also a recent survey on the appearance of convective rolls [12] and the references in it. It should be remarked that in all the enumerated works corrections on temperature gradient were not taken into account, i. e. the temperature entering into the intensity of the fluctuating forces was assumed to be equal to some average.

The application of the Langevin method with the Landau-Lifshitz fluctuating forces to hydrodynamic instabilities has led to the creation of the whole physical branch named fluctuation hydrodynamics. The adherents of fluctuation hydrodynamics either kept silent as regards the fact that Einstein's and Langevin's approaches gives varying results for fluctuations in the RayleighBenard problem or explained that Einstein's approach cannot be used for the description of nonequilibrium fluctuations.

And only recently by the formal use of the fluctuationdissipation theorem (FDT) [13] it was shown that the cause of different results given by Einstein's and Langevin's methods is coupled with the fact that for the convective Rayleigh-Benard instability fluctuating forces are distinguished with the cross-term's presence from the Landau-Lifshitz fluctuating forces. In doing so, the basic assumption of works [3, 6-12] about the independence of the fluctuating forces on the proximity of the stability threshold turns out to be incorrect: the fluctuating forces depend on the proximity of the threshold, and fluctuations calculated on this basis behave themselves as it was predicted in [4]. 
In the present work the corresponding formulas (FDT) will be obtained directly from the input equations. This is a more convenient way for the demonstration of equivalence of Einstein's and Langevin's methods. Besides, using Langevin's description, the change of the temperature of the liquid layer will be taken into account and the peculiarities of the fluctuating forces of the unstable mode will be discussed.

The basis of the subsequent exposition is the localequilibrium. The Navier-Stokes equations describing the hydrodynamic instabilities are true in this assumption. A deviation from a local-equilibrium would lead to the examination of more complex equations than the hydrodynamic ones. In general there is no need to attract the kinetic theory for the description of the hydrodynamic instabilities: the intensity of the fluctuating forces should be found on the hydrodynamic level. At the same time the consideration of the long wave effects coupled with the temperature gradient reduces to the account of the space dispersion of the local-equilibrium distribution and may be easily realized.

\section{TWO METHODS OF FLUCTUATION DESCRIPTION IN HYDRODYNAMICS}

Two opportunities exist to describe hydrodynamic fluctuation. The first one may be called Einstein's (or Einstein-Onsager's) approach. It is based on the fact that at the initial moment of time fluctuation distribution is supposed to be known and the subsequent evolution of fluctuations is described by macroscopic equations of hydrodynamics.

The second approach i. e. the Langevin one consists in the fact that the input evolution equations are written down with the fluctuating forces. The correlation functions of the fluctuating forces should be chosen so that both the approaches could give equal results for the correlation functions of fluctuations.

As a matter of fact, Einstein's and Langevin's approaches are two methods of the solution in the general case of the set of differential equations either homogeneous with the random initial condition defined by the corresponding distribution function, or heterogeneous, so that the random heterogeneity is the source of the fluctuation perturbations. The results of the solution should be equal for any moment of time and at the initial moment, in particular. This permits to find the intensity of fluctuating forces on the following recipe, which we shall demonstrate for the simplest system, fluctuations in which are described by the equation:

$$
\dot{x}=-\lambda x
$$

and the distribution function of initial fluctuation is Gaussian:

$$
f(x) \propto \exp \left(-\frac{1}{2} \beta x^{2}\right) .
$$

Let us add to the right part of (1) the fluctuating force $y$, for which

$$
\langle y(\tau) y(0)\rangle=Q \delta(\tau)
$$

With the fluctuating force the solution of (1) takes the form

$$
x=e^{-\lambda t} \int_{-\infty}^{t} y(\tau) e^{\lambda \tau} d \tau
$$

Putting $t=0$, squaring and averaging we get the wellknown Einstein's formula for the intensity of the fluctuating forces:

$$
Q=2 \lambda \beta^{-1}
$$

Formula (5), in which $\beta^{-1}$ determines the single-time correlation function $\left\langle x^{2}\right\rangle$, is the most simple FDT, which expresses the intensity or spectral density of the fluctuating forces through the evolution parameter $\lambda$ and the single-time correlation function.

Below we shall apply this method for the finding of the fluctuating forces in the Rayleigh-Benard problem by preliminary defining single-time correlation functions.

\section{SINGLE-TIME CORRELATION FUNCTIONS OF FLUCTUATION IN THE FLUID LAYER WITH TEMPERATURE GRADIENT}

The input equations describing the Rayleig-Benard instability of the horizontal liquid layer with the thickness $l$ and free boundaries in the gravitation field in the Boussinesq approximation [14] have the form

$$
\begin{aligned}
& \frac{\partial \vec{\nu}}{\partial t}=-\frac{1}{\rho} \vec{\nabla} p+\nu \Delta \vec{\nu}-\alpha \vec{g} \theta, \\
& \frac{\partial \theta}{\partial t}+\vec{\nu} \vec{\nabla} T=\chi \Delta \theta \\
& \operatorname{div} \vec{\nu}=0
\end{aligned}
$$

with the boundary conditions for $z=0, l$ :

$$
w=0, \quad \frac{\partial \nu_{x}}{\partial z}=\frac{\partial \nu_{y}}{\partial z}=0, \quad \theta=0
$$

Here $\vec{\nu}\left(\nu_{x}, \nu_{y}, w\right), \theta, p$ are the velocity, temperature and pressure perturbations, $\vec{\nabla} T=-\beta \vec{z}^{0}$ is the vertical gradient of the temperature, $\nu$ is the kinematic viscosity, $\alpha$ is the thermal expansion coefficient, $\chi$ is the thermal diffusivity, $\vec{g}$ is the acceleration of gravity, $\rho$ is the density of the fluid. 


\section{P. LESNIKOV}

The boundary conditions allow us to search for the solution of the problem in the expansion form

$$
\left(\begin{array}{c}
w \\
\theta
\end{array}\right)=\sqrt{\frac{2}{V}} \sum_{\vec{k}}\left(\begin{array}{c}
w_{\vec{k}} \\
\theta_{\vec{k}}
\end{array}\right) e^{i \overrightarrow{\mathrm{K}} \vec{r}} \sin q z,
$$

where $\overrightarrow{\mathrm{K}}$ and $\vec{r}$ lie in the horizontal plane, $q=n \pi / l, n=$ $1,2, \ldots, \vec{k}=\overrightarrow{\mathrm{K}}+\vec{q}, V$ is the volume of the layer. Analogous expansions take place for $v_{x}, v_{y}$ with the substitution of sine for cosine.

Local-equilibrium distribution function of the initial fluctuations is defined by kinetic and intrinsic fluctuations energy:

$$
f(\vec{\nu}, \theta) \propto \exp \left(-\int\left(\frac{\rho \vec{\nu}^{2}}{2 T}+\frac{c_{V} \theta^{2}}{2 T^{2}}\right) d V\right)
$$

where $c_{V}$ is the specific heat, integration is carried out

all over the volume, Boltzmann's constant is omitted and the temperature is changed from the of the lower boundary $T_{1}$ to the temperature of the upper boundary $T_{2}$ according to the law

$$
T=T_{1}-\beta z \text {. }
$$

Let us insert into (9) expansions of fluctuation perturbations and (10). Then taking into account the incompressibility condition and retaining linear terms to $\beta$ when integrating, we get the following correlation functions for the quantities $\theta_{\vec{k}}, w_{\vec{k}}, \nu_{x \vec{k}}, \nu_{y \vec{k}}$ :

$$
\begin{aligned}
\left\langle\theta_{\vec{k}} \theta_{\vec{k}^{\prime}}\right\rangle & =\left(\frac{T_{1} T_{2}}{c_{V}} \delta_{q, q^{\prime}}+\frac{4 A T_{1} q q^{\prime}}{c_{V}} \Delta_{q, q^{\prime}}\right) \delta_{\overrightarrow{\mathrm{K}},-\overrightarrow{\mathrm{K}}}, \\
\left\langle w_{\vec{k}} w_{\vec{k}^{\prime}}\right\rangle & =\left(\frac{\bar{T} \mathrm{~K}^{2}}{\rho k^{2}} \delta_{q, q^{\prime}}+\frac{A \mathrm{~K}^{2} q q^{\prime}}{\rho k_{*}^{2}} \Delta_{q, q^{\prime}}\right) \delta_{\overrightarrow{\mathrm{K}},-\overrightarrow{\mathrm{K}}^{\prime}},
\end{aligned}
$$

where $\alpha=x, y, k_{*}^{-2}=k^{-2}+k^{-2}, \bar{T}=\left(T_{1}+T_{2}\right) / 2, \delta$ with indices is the Kroneker's symbol, $\Delta_{q, q^{\prime}}$ is equal to one if $q$ and $q^{\prime}$ are different multiples of $\pi / l$ and zero in the rest of the cases,

$$
A=\frac{4 \Delta T}{l^{2}\left(q^{2}-q^{2}\right)^{2}} .
$$

The appearance of the imaginary unit in (14) is connected with the phase shift in the expansions $v_{x}, v_{y}$ and $w$.

The possibility of the expansion on $\beta$ in (9) caused by the fact that hydrodynamics is restricted by the region of small gradients of macroscopic fields of velocity, temperature, etc. In our case the stated expansion signifies small difference of temperatures of the boundaries of the layer $\Delta T$ in comparison with the temperatures of the boundaries themselves and guarantees the smallness of the corresponding corrections in comparison with the equilibrium approximation.

In the experiments on the convective instability carried out at a room temperature of the liquid layer the temperature difference at the moment of appearance of the instability does not exceed $5^{\circ} \mathrm{C}$.In the percent relation this is less than $2 \%$. So for the silicon oil the working temperature is $22^{\circ} \mathrm{C}$ and the critical difference of the temperature is $4.51^{\circ} \mathrm{C}$ [15].

On account of this, the equilibrium approximation is the basic one. It keeps space homogeneity of the problem leading to the symmetry on the frequency of the fluctuation spectra. Corrections destroy this homogeneity and it will be shown that they give non-symmetry of the spectra just as it takes place for the fluctuations in the unlimited liquid with the temperature gradient $[16$, 17]. As to Hinton's kinetic corrections they represent the kinetic way out of the hydrodynamic limits and cannot give such long-wave peculiarities.

\section{FLUCTUATING FORCES AND SPECTRA OF FLUCTUATIONS IN THE EQUILIBRIUM APPROXIMATION}

Equations (6) may be transformed in equations only for two variables: the vertical component of velocity $w$ 
and the temperature $\theta$ :

$$
\begin{aligned}
& \Delta \frac{\partial w}{\partial t}=\nu \Delta^{2} w+\alpha g\left(\frac{\partial^{2}}{\partial x^{2}}+\frac{\partial^{2}}{\partial y^{2}}\right) \theta \\
& \frac{\partial \theta}{\partial t}=\beta w+\chi \Delta \theta
\end{aligned}
$$

From here for the values of $w_{\vec{k}}, \theta_{\vec{k}}$ we get

$$
\begin{aligned}
& \dot{w}=-\nu w+\alpha \theta, \\
& \dot{\theta}=\beta w-\chi \theta,
\end{aligned}
$$

where redesignations are carried out

$$
\nu k^{2} \rightarrow \nu, \alpha g \frac{\mathrm{K}^{2}}{k^{2}} \rightarrow \alpha, \chi k^{2} \rightarrow \chi
$$

and here and later on the index $\vec{k}$ will be omitted.

The eigenvalues of system (18) are equal to

$$
-\Omega_{ \pm}=-\frac{1}{2}(\nu+\chi \pm E)
$$

where

$$
E=\sqrt{(\nu-\chi)^{2}+4 \alpha \beta} .
$$

The evident properties are fulfilled for them:

$$
\Omega_{+}+\Omega_{-}=\nu+\chi, \Omega_{+} \Omega_{-}=\nu \chi-\alpha \beta
$$

The Rayleigh-Benard instability comes when $\Omega_{-}$vanishes. The gradient of the temperature is such that till this moment

$$
\nu \chi-\alpha \beta=0
$$

Condition (23) determines the critical meaning of the adjustable parameter $\beta$ or its dimensionless analog Rayleigh number $R=\frac{\alpha \beta g l^{4}}{\nu \chi}$. For the unstable mode, as is well known, $q=\pi / l, \mathrm{~K}=\pi / \sqrt{2} l$ and $R=27 \pi^{4} / 4$.

In equilibrium approximation the single-time correlation functions are equal to

$$
\left\langle|w|^{2}\right\rangle=\frac{\bar{T} \mathrm{~K}^{2}}{\rho k^{2}},\langle w \theta\rangle=0,\left\langle|\theta|^{2}\right\rangle=\frac{\bar{T}^{2}}{c_{V}}
$$

Let us add to the right parts of equations (18) the fluctuating forces $f_{1}$ and $f_{2}$. By virtue of the stationary and homogeneity of the considered approximation their correlation functions should be sought in the form

$$
\left\langle f_{i}(\tau) f_{j}^{\prime}(0)\right\rangle=Q_{i j} \delta_{q, q^{\prime}} \delta_{\overrightarrow{\mathrm{K}},-\overrightarrow{\mathrm{K}}^{\prime}} \delta(\tau)
$$

where $Q_{i j}$ is the symmetric matrix and dash in $f_{j}^{\prime}$ denotes here and further that the corresponding value is defined for $\vec{k}^{\prime}$.

Let us write down the solution of equations (18) at the presence of the fluctuating forces:

$$
\left(\begin{array}{c}
w \\
\theta
\end{array}\right)=\left(\begin{array}{c}
1 \\
\frac{\nu-\Omega_{+}}{\alpha}
\end{array}\right) e^{-\Omega_{+} t} \int_{-\infty}^{t}\left[f_{1}\left(\nu-\Omega_{-}\right)-\alpha f_{2}\right] e^{\Omega_{+} \tau} d \tau+\left(\begin{array}{c}
1 \\
\frac{\nu-\Omega_{-}}{\alpha}
\end{array}\right) e^{-\Omega_{-} t} \int_{-\infty}^{t}\left[-f_{1}\left(\nu-\Omega_{-}\right)+\alpha f_{2}\right] e^{\Omega_{-} \tau} d \tau
$$

Putting $t=0$ in (26) and then creating square terms, we get the following set of equations for the definition of $Q_{i j}$ :

$$
\begin{aligned}
& \left\langle|w|^{2}\right\rangle=\frac{\left(\chi^{2}+\Omega_{+} \Omega_{-}\right) Q_{11}+2 \alpha \chi Q_{12}+\alpha^{2} Q_{22}}{2 \Omega_{+} \Omega_{-}\left(\Omega_{+}+\Omega_{-}\right)}, \\
& 0=\beta \chi Q_{11}+2 \nu \chi Q_{12}+\alpha \nu Q_{22} \\
& \left\langle|\theta|^{2}\right\rangle=\frac{\beta^{2} Q_{11}+2 \beta \nu Q_{12}+\left(\nu^{2}+\Omega_{+} \Omega_{-}\right) Q_{22}}{2 \Omega_{+} \Omega_{-}\left(\Omega_{+}+\Omega_{-}\right)} .
\end{aligned}
$$

From (27) we find

$$
\begin{aligned}
& Q_{11}=2 \nu\left\langle|w|^{2}\right\rangle, \\
& Q_{12}=-\beta\left\langle|w|^{2}\right\rangle-\alpha\left\langle|\theta|^{2}\right\rangle, \\
& Q_{22}=2 \chi\left\langle|\theta|^{2}\right\rangle .
\end{aligned}
$$

The obtained formulas are in conformity with the formal record of FTD, which has the form

$$
Q_{i j}=\gamma_{i j}+\gamma_{j i}, \gamma_{i j}=\lambda_{i k} \beta_{k j}^{-1}
$$

for the so called Orshtein-Uhlenbeck process [17] defined by the set of differential equations with the distribution 


\section{P. LESNIKOV}

function of the initial fluctuations

$$
\dot{x}_{i}=-\lambda_{i k} x_{k}, f(\vec{x}) \propto \exp \left(-\frac{1}{2} \beta_{i k} x_{i} x_{k}\right) .
$$

The decisive difference from the work [3] and the subsequent works is the presence of the cross correlations in (28), which change all inferences of these works concerning fluctuations near the convective threshold.

Cross correlations violate the Onsager principle $\left(\gamma_{12}, \gamma_{21} \neq 0\right)$. This is connected with the loss of the property of definite evennes by the functions $w$ and $\theta$. Every one of them (see below (43),(44)) depends on both the initial conditions of $w(0)$ and $\theta(0)$. When we inverse the time $w(0)$ changes and $\theta(0)$ does not changes the sign. That is why $w$ and $\theta$ are neither odd nor even.

The explicit from the matrix $\gamma_{i j}$ is equal to

$$
\gamma_{i j}=\left(\begin{array}{cc}
\nu\left\langle|w|^{2}\right\rangle & -\alpha\left\langle|\theta|^{2}\right\rangle \\
-\beta\left\langle|w|^{2}\right\rangle & \chi\left\langle|\theta|^{2}\right\rangle
\end{array}\right)
$$

The conversion to the zero of the determinant $\left|\gamma_{i j}\right|$ at the instability point is an important property. This also directly follows from the definition of $\gamma_{i j}$ and the conversion to the zero of the determinant $\left|\lambda_{i j}\right|$ for the null root of the dispersion equation $\left|-i \omega \delta_{i j}+\lambda_{i j}\right|=0$.

Let us define the spectrum of the fluctuating value $\phi(\vec{r}, z, t)$ as Fourier transformation on space and time of its two-time correlation function:

$$
\langle\phi(2) \phi(1)\rangle_{\vec{k}, \omega}=\int_{-\infty}^{\infty} \int_{-\infty}^{\infty} \int_{V} \int_{V}\langle\phi(2) \phi(1)\rangle^{i \omega\left(t_{2}-t_{1}\right)-i \vec{k}\left(\vec{r}_{2}-\vec{r}_{1}\right)-i q\left(z_{2}-z_{1}\right)} d t_{1} d t_{2} d \vec{r}_{1} d \vec{r}_{2} d z_{1} d z_{2}
$$

where we omit the multiplier connected with the volume and experiment duration, and the set of space-time coordinates is denoted here by the figures in arguments.

Inserting into (32) expansion (8) we get

$$
\langle\phi(2) \phi(1)\rangle_{\vec{k}, \omega}=\sum_{q^{\prime \prime}, q^{\prime}}\left\langle\phi_{\omega}^{\prime \prime} \phi_{-\omega}^{\prime}\right\rangle I\left(q^{\prime \prime}, q^{\prime}, q\right)
$$

where

$$
\begin{aligned}
& I\left(q^{\prime \prime}, q^{\prime}, q\right)= \\
& =\frac{4}{l^{2}} \int_{0}^{l} \int_{0}^{l} e^{-i q\left(z_{2}-z_{1}\right)} \sin q^{\prime} z_{1} \sin q^{\prime \prime} z_{2} d z_{1} d z_{2}
\end{aligned}
$$

two dashes denote the dependence on $\left(\overrightarrow{\mathrm{K}}, q^{\prime \prime}\right)$ and dash that on $\left(-\overrightarrow{\mathrm{K}}, q^{\prime}\right)$.
In the equilibrium approximation $q^{\prime}=q^{\prime \prime}$.Besides, we shall confine ourselves to $q$, which is a multiple of $\pi / l$, then the greatest contribution gives the term with $q^{\prime}=q^{\prime \prime}=q$, the module of both the integrals in (34) for which is equal to $l / 2$, and $I=1$.

In consequence using the solution of equations (18) with added fluctuating forces

$$
\begin{gathered}
w_{\omega}=\left[(-i \omega+\chi) f_{1 \omega}+\alpha f_{2 \omega}\right] / \Delta, \\
\theta_{\omega}=\left[\beta f_{1 \omega}+(-i \omega+\nu) f_{2 \omega}\right] / \Delta,
\end{gathered}
$$

where

$$
\Delta=\left(-i \omega+\Omega_{+}\right)\left(-i \omega+\Omega_{-}\right)
$$

and the expressions for the fluctuating forces $(25),(28)$, we get

$$
\begin{aligned}
& \langle w(2) w(1)\rangle_{\vec{k}, \omega}=2\left\langle|w|^{2}\right\rangle\left[\left(\frac{\nu-\Omega_{-}}{E}\right) \frac{\Omega_{+}}{\omega^{2}+\Omega_{+}^{2}}+\left(\frac{\chi-\Omega_{-}}{E}\right) \frac{\Omega_{-}}{\omega^{2}+\Omega_{-}^{2}}\right], \\
& \langle\theta(2) \theta(1)\rangle_{\vec{k}, \omega}=2\left\langle|\theta|^{2}\right\rangle\left[\left(\frac{\chi-\Omega_{-}}{E}\right) \frac{\Omega_{+}}{\omega^{2}+\Omega_{+}^{2}}+\left(\frac{\nu-\Omega_{-}}{E}\right) \frac{\Omega_{-}}{\omega^{2}+\Omega_{-}^{2}}\right],
\end{aligned}
$$

thus, every of the fluctuation spectra presents itself two Lorentz lines corresponding to fast stable $\left(\Omega_{+}\right)$and slow unstable $\left(\Omega_{-}\right)$modes. 
With the approach to the instability

$$
E, \Omega_{+} \rightarrow \nu+\chi, \Omega_{-} \rightarrow 0
$$

and the singular contributions appear in spectra:

$$
\begin{aligned}
& \langle w(2) w(1)\rangle_{\vec{k}, \omega}=2\left\langle|w|^{2}\right\rangle\left[\frac{\nu}{\omega^{2}+(\nu+\chi)^{2}}+\frac{\chi}{\nu+\chi} \pi \delta(\omega)\right], \\
& \langle\theta(2) \theta(1)\rangle_{\vec{k}, \omega}=2\left\langle|\theta|^{2}\right\rangle\left[\frac{\nu}{\omega^{2}+(\nu+\chi)^{2}}+\frac{\chi}{\nu+\chi} \pi \delta(\omega)\right] .
\end{aligned}
$$

The same results will be obtained if one writes down the solution of equations (18) with the initial conditions

$$
\begin{aligned}
w(t) & =\frac{1}{E}\left\{w(0)\left[\left(\nu-\Omega_{-}\right) e^{-\Omega_{+} t}+\left(\chi-\Omega_{-}\right) e^{-\Omega_{-} t}\right]\right. \\
& \left.-\alpha \theta(0)\left(e^{-\Omega_{+} t}-e^{-\Omega_{-} t}\right)\right\}, \\
\theta(t) & =\frac{1}{E}\left\{-\beta w(0)\left(e^{-\Omega_{+} t}-e^{-\Omega_{t}}\right)\right. \\
& \left.+\theta(0)\left[\left(\chi-\Omega_{-}\right) e^{-\Omega_{+} t}+\left(\nu-\Omega_{-}\right) e^{-\Omega_{-} t}\right]\right\}
\end{aligned}
$$

and does averaging and the Fourier trasformation in time.

Therefore, both Einstein's and Langevin's approaches give equivalent results in the basic equilibrium approximation when correct definition of fluctuating forces is made. And it is natural, inasmuch as we speak about the two methods of the solution of the same mathematical problem.

The formulas (38), (39), (41), (42) were obtained in [4] where spectra were defined with the multiplier $(2 \pi)^{-1}$. It is only necessarily to omit kinetic corrections and to ignore the compressibility.

\section{INFLUENCE OF DISPERTION OF THE TEMPERATURE ON THE FLUCTUATION SPECTRA}

The problem of finding fluctuation spectra, when the temperature gradient is present in the unlimited liquid, primarily was considered by rather complicated methods [18-20] and only then it was noticed that all peculiarities caused by the temperature gradient one can get by the Langevin way, taking into account the dependence of the temperature on coordinates in the intesity of the fluctuating forces $[21,22,16]$. It just means the account of the temperature dispersion in the approximation of a local equilibrium.

The distinctive feature of the similar problem for the convective instability is the presence of the boundaries leading to discrete band of the modes, the single-time correllation functions of which are defined by formulas (11)-(16).

Corresponding expressions for the correlation functions of fluctuating forces are received by the method stated above:

$$
\left\langle f_{i}(\tau) f_{j}^{\prime}(0)\right\rangle=\left(\begin{array}{cc}
\left(\nu+\nu^{\prime}\right)\left\langle w w^{\prime}\right\rangle & -\beta\left\langle w w^{\prime}\right\rangle-\alpha\left\langle\theta \theta^{\prime}\right\rangle \\
-\beta\left\langle w w^{\prime}\right\rangle-\alpha^{\prime}\left\langle\theta \theta^{\prime}\right\rangle & \left(\chi+\chi^{\prime}\right)\left\langle\theta \theta^{\prime}\right\rangle
\end{array}\right) \delta(\tau) .
$$

Formula (45) represents FTD taking into account the temperature change in the local-equilibrium distribution function. With its help from (33) for the spectrum of the temperature fluctuations we get

$$
\langle\theta(2) \theta(1)\rangle_{\vec{k}, \omega}=\sum_{q^{\prime \prime}, q^{\prime}}\left\langle\theta^{\prime \prime} \theta^{\prime}\right\rangle \frac{\left(i \omega+\nu^{\prime}\right) \Omega_{+}^{\prime \prime} \Omega_{-}^{\prime \prime}+\left(-i \omega+\nu^{\prime \prime}\right) \Omega_{+}^{\prime} \Omega_{-}^{\prime}+\omega^{2}\left(\chi^{\prime}+\chi^{\prime \prime}\right)}{\left(-i \omega+\Omega_{-}^{\prime \prime}\right)\left(-i \omega+\Omega_{+}^{\prime \prime}\right)\left(i \omega+\Omega_{-}^{\prime}\right)\left(i \omega+\Omega_{+}^{\prime}\right)} I\left(q^{\prime \prime}, q^{\prime}, q\right) .
$$

The expansion on elementary fractions gives

$$
\langle\theta(2) \theta(1)\rangle_{\vec{k}, \omega}=\sum_{q^{\prime \prime}, q^{\prime}}\left\langle\theta^{\prime \prime} \theta^{\prime}\right\rangle\left(D_{\omega}^{\prime \prime}+D_{-\omega}^{\prime}\right) I\left(q^{\prime \prime}, q^{\prime}, q\right),
$$




\section{P. LESNIKOV}

where

$$
D_{\omega}=\left(\frac{\chi-\Omega_{-}}{E}\right) \frac{1}{-i \omega+\Omega_{+}}+\left(\frac{\nu-\Omega_{-}}{E}\right) \frac{1}{-i \omega+\Omega_{-}} .
$$

Let us note, that we also obtain (47) at once, if we use Einstein's approach.

For $q$, which is a multiple of $\pi / l$, the essential contribution in sum (47) except the term with $q^{\prime}=q^{\prime \prime}=q$ give the terms at which either $q^{\prime}=q$ or $q^{\prime \prime}=q$. For them a module of one of the integrals entering in (34) equals $l / 2$ and

$$
I\left(q, q^{\prime}, q\right)=I^{*}\left(q^{\prime}, q, q\right)=\frac{4 i q^{\prime}}{l\left(q^{2}-q^{\prime 2}\right)}
$$

The spectrum of the temperature fluctuations consists of the already written results of the basic equilibrium approximation where we must change $\bar{T}^{2}$ in $\left\langle|\theta|^{2}\right\rangle$ on $T_{1} T_{2}$ according to the first term (11). A correction linked with the second term in (11) also appears.

$$
\Delta\langle\theta(2) \theta(1)\rangle_{\vec{k}, \omega}=-\frac{128 \Delta T T_{1} q}{c_{V} l^{3}} \sum_{q^{\prime}} \frac{q^{\prime 2}}{\left(q^{2}-q^{\prime 2}\right)^{3}} \operatorname{Im}\left(D_{\omega}+D_{-\omega}^{\prime}\right) .
$$

The summation in (50) is over $q^{\prime}$, which has the evenness opposite to $q$. The imaginary part of $D_{\omega}$ in accordance with (48) is proportional to $\omega$, that is why (50) is odd frequency function.

The presence in the denominator of (50) of the value $\left(q^{2}-q^{\prime 2}\right)^{3}$ allows when summed to be confined to the only terms with $q^{\prime}$ differing from $q$ on $\pi / l$ which corresponds to the accounting of the correlation among the neighbouring modes. If also one suggests that $q>>\pi / l$, then formula (50) can be represented in the form

$$
\Delta\langle\theta(2) \theta(1)\rangle_{\vec{k}, \omega}=-\frac{32 \Delta T T_{1}}{c_{V} \pi^{2} l} \frac{\partial}{\partial q} \operatorname{Im} D_{\omega}
$$

or in the explicit form

$$
\begin{aligned}
\Delta\langle\theta(2) \theta(1)\rangle_{\vec{k}, \omega} & =\frac{64 \Delta T T_{1} q \omega}{k^{2} l \pi^{2} c_{V}}\left\{\frac{\alpha \beta-2 \nu^{2}}{\left(\omega^{2}+\Omega_{+}^{2}\right)\left(\omega^{2}+\Omega_{-}^{2}\right)}\right. \\
& \left.+\frac{2\left[\omega^{2}\left(\nu^{2}+\chi^{2}-\alpha \beta\right)+\Omega_{+} \Omega_{-}(2 \nu \chi+\alpha \beta)\right]\left(\omega^{2}+\nu^{2}+\alpha \beta\right)}{\left(\omega^{2}+\Omega_{+}^{2}\right)^{2}\left(\omega^{2}+\Omega_{-}^{2}\right)^{2}}\right\} .
\end{aligned}
$$

Analogous corrections can be written for the spectra of velocity.

\section{FLUCTUATING FORCES FOR THE UNSTABLE MODE}

Let us define the fast and the slow mode by formulas

$$
x_{ \pm}=w+\left(\nu-\Omega_{ \pm}\right) \theta / \beta .
$$

The evolution of these modes is described by the equations

$$
\dot{x}_{ \pm}=-\Omega_{ \pm} x_{ \pm}
$$

Fluctuating forces for equations (54) $\eta_{ \pm}$are expressed in $f_{1}$ and $f_{2}$ as well as in accordance with (53) $x_{ \pm}$are expressed in $w$ and $\theta$.
If the intensity of the fluctuating forces $\eta_{-}$is calculated by using formulas by Landau and Lifshitz, then

$$
Q=2 \nu\left\langle|w|^{2}\right\rangle+2 \chi\left\langle\theta^{2}\right\rangle\left(\frac{\nu-\Omega_{-}}{\beta}\right)^{2}
$$

and the intesity of the fluctuating forces slightly changes with the approach of the threshold of stability remaining finite in the point of stability itself. Then the FokkerPlank equation for the slow mode distribution function:

$$
\frac{\partial f}{\partial t}=\frac{\partial}{\partial x_{-}}\left(\Omega_{-} x_{-} f\right)+\frac{1}{2} Q \frac{\partial^{2} f}{\partial x_{-}^{2}}
$$

in the stationary case has the solution: 


$$
f_{s t} \propto \exp \left(-\frac{\Omega_{-} x_{-}^{2}}{Q}\right)
$$

It follows, taking into account the terms of higher order, the analogy with phase transition of the second order and a divergency of single-time correlation functions with the approach to the point of stability when $\Omega_{-} \rightarrow 0$.

In reality relations $(25),(28)$ give

$$
Q=2 \Omega_{-}\left\langle x_{-}^{2}\right\rangle
$$

where

$$
\left\langle x_{-}^{2}\right\rangle=\left\langle|w|^{2}\right\rangle+\left(\frac{\nu-\Omega_{-}}{\beta}\right)^{2}\left\langle|\theta|^{2}\right\rangle .
$$

From (58) it follows that the intensity of the fluctuating forces $Q$ of slow mode tends to zero with $\Omega_{-}$; stationary distribution function does not feel any singularities and the fluctuations $x_{-}$remain on the ordinary level. At the same time, as it is easy to understand, the dissipation function of slow mode vanishes.

In this convective Reileigh-Benard instability does not differ from the instability of the oscillator caused by diminishing damping or from that of the oscillatory circuit with diminishing resistance. In both cases according to the formulas offered by Einstein and Naiquist the intensity of the fluctuating forces vanishes.

The generalisation of formula (58) in the case of temperatures dispersion is

$$
\left\langle\eta_{-}(\tau) \eta_{-}^{\prime}(0)\right\rangle=\left(\Omega_{-}+\Omega_{-}^{\prime}\right)\left\langle x_{-} x_{-}^{\prime}\right\rangle \delta(\tau)
$$

which leads to longwave tail in spectrum as described above.

\section{SUMMARY}

Thus, in the present paper it is shown that hydrodynamic theory of thermal fluctuations must be based on the approximation of the local equilibrium; for convective instability equilibrium approximation is basic by virtue of the smallness of the temperature drop $\Delta T$ in comparison with the temperature of the boundaries themselves; the correlations between different fluctuation modes when the temperature gradient presence leads to the asymmetry of the of the spectra; fluctuating forces depend on the proximity of the threshold of stability, in particular, the intensity of the fluctuating forces of the unstable mode vanishes in the point of instability; integrated intensities of the fluctuations do not have any singularities; nonlimited narrowing of the line corresponding to the unstable mode takes place only in the fluctuation spectra.

The boundaries of such behaviour of the thermal fluctuations were stated in [4]. It is true in the whole region of the Rayleigh number change excluding the extremely narrow interval $\Delta R$ near the instability point.

In the experimental work [23] it was reported about the chaotic character of the appearance of the RayleighBenard instability. Furthermore it was found that in accordance with the experimental data the intensity of the fluctuating forces causing the chaos must be on four-six orders larger than the value following from the thermal estimates on the basis of the Landau-Lifshitz fluctuating forces. As we pointed here the use of the Landau-Lifshitz fluctuating forces to the Rayleigh-Benard instability is wrong. So the explanation of the chaotic character of the appearance of the Rayleigh-Benard instability must be sought elsewhere.
[1] Г. Уленбек, УФН 103, 275 (1971).

[2] Л. Д. Ландау, Е. М. Лифшиц, ЖЭТФ 32, 618 (1957).

[3] В. М. Зайцев, М. И. Шлиомис, ЖЭТФ 59, 1583 (1970).

[4] В. П. Лесников, И. З. Фишер, ЖЭТФ 67, 1341 (1974).

[5] F. L. Hinton, Phys. Fluids 13, 857 (1970).

[6] R. Graham, Phys. Rev. A10, 1762 (1974).

[7] J. Swift, and P. C. Hohenberg, Phys. Rev. A15, 319 (1977).

[8] H. Haken, Sinergetics (Springer-Verlag, Berlin, Heilderberg, NY, 1978).

[9] Y. Tsuhiya, Phys. Soc. Jap. 43, 1823 (1977).

[10] H. N. W. Lekkerkerker, J. -P. Boon, in Fluctuat., instabil., and Phase Transit. (ed. by T. Rise, NY, London, 1975).

[11] H. van Beijeren, E. G. D. Cohen, J. Stat. Phys. 53, 285 (1988).

[12] M. C. Cross, P. C. Hohenberg, Rev. Mod. Phys. 65, 851 (1993).
[13] В. П. Лєсніков, Укр. фіз. журн. 39, 379 (1994); 41, 181 (1996).

[14] Л. Д. Ландау, Е. М. Лифшиц, Гидродинамика (Наука, Москва, 1986).

[15] J. Wesfreid, Y. Pomeau, M. Dubois, C. Normand, P. Berge, J. Phys. (France) Lett. 39, 725 (1978).

[16] A. -M. S. Tremblay, M. Arai, E. D. Siggia, Phys. Rev. A23, 1451 (1981).

[17] J. Kazer, Statistical Thermodinamics of Nonequilibrium Processes (Springer-Verlag, Berlin, Heildenberg, London, Paris, Tokyo, 1987).

[18] I. Proccacia, D. Ronis, M. A. Collins, J. Ross, I. Oppenheim, Phys. Rev. A19, 1290 (1979).

[19] I. Proccacia, D. Ronis, I. Oppenheim, Phys. Rev. A19, 1307 (1979); Phys. Rev. A19, 1324 (1979).

[20] T. Kirkpatrick, E. G. Cohen, J. R. Dorfman, Phys. Rev. Lett. 42, 862 (1979).

[21] G. van der Zwan, P. Mazur, Physics Lett. 75A, 370 
$(1980)$.

[22] D. Ronis, S. Patterman, Phys. Rev. A22, 773 (1980).
[23] C. W. Meyer, G. Ahlers, D. S. Cannell, Phys. Rev. Lett. 59, 1577 (1987).

\section{ТЕПЛОВІ ГІДРОДИНАМІЧНІ ФЛЮКТУАЩЇ̈ ПОБЛИЗУ НЕСТІЙКОСТІ РЕЛЕЯ-БЕНАРА}

В. П. Лєсніков

Одесъкий державний політехнічний університет, кафедра фізики

Украӥна, UA-270044, Одеса

Показана еквівалетність опису гідродинамічних флуктуацій айнштайнівським та ланжевенівським підходами в задачі Релея-Бенара. Вказано на помилковість аналогії, заснованої на використанні сил ЛандауЛіфшица до конвективної нестійкості з фазовими переходами. 